\title{
Intervención educativa tras el terremoto 2017: comprendiendo procesos identitarios en mujeres rurales
}

\section{Educational intervention after 2017's earthquake: Understanding identity processes in rural women}

\author{
María del Pilar Ramírez VARElA*
}

\begin{abstract}
En este artículo se presentan los avances de una investigación e intervención educativa cuyo objetivo es la comprensión de los procesos identitarios en las mujeres de una comunidad rural apoyada después del terremoto de 2017. Durante más de dos años se trabajó con mujeres que aceptaron integrarse a un grupo de apoyo. En las sesiones quincenales se plantearon situaciones lúdicas y reflexivas que fueron haciendo que las participantes se reconocieran desde su valor personal, compartieran las experiencias de sus historias particulares y de su cotidianidad, se vincularan entre ellas para tomar decisiones y construir nuevas experiencias que, a su vez, fueron favoreciendo nuevas formas de identidad individual y colectiva.
\end{abstract}

This article presents the advances in the educational research and intervention. The objective is to understand the identity processes in the women of the community throughout and after the earthquake. For more than two years, we worked with women who voluntarily agreed to join a support group. In biweekly sessions, recreational and reflexive situations were raised that caused the participants to recognize themselves on their personal value, to share the experiences of their particular stories and their daily lives, to link with each other and make decisions in order to build new experiences that help new forms of individual and collective identity.

\section{Palabras clave:}

vulnerabilización, intervención educativa, mujeres rurales, procesos identitarios

\section{Keywords:}

vulnerability, educative intervention, rural women, identity process

Recibido: 20 de febrero de 2020 | Aceptado para su publicación: 23 de septiembre de 2020 | Fecha de publicación: 1 de octubre de 2020

Recuperado de: https://sinectica.iteso.mx/index.php/SINECTICA/article/view/1118 doi: 10.31391/S2007-7033(2020)0055-009

*Doctoranda en Investigación e Intervención Educativa por la Universidad Pedagógica Nacional. Coordinadora de Capacitación del Instituto de Terapias Integrales y miembro del Instituto Nacional de Investigación en Desarrollo Humano. Líneas de investigación: proyectos locales, autonomía educativa, desarrollo comunitario y género. Correo electrónico: piliramirezvarela@hotmail.com/https://orcid.org/0000-0002-6912-1711 


\section{INTRODUCCIÓN}

Araíz del terremoto del 19 de septiembre de 2017, muchas poblaciones ruA rales del estado de Morelos sufrieron daños. En la comunidad de Valle de Vázquez, Tlaquiltenango, de las 394 viviendas que existían, según datos del Consejo Nacional de Población, 101 fueron dañadas de una u otra manera (Censo de Damnificados, Ayudantía Municipal). También, hubo destrucción parcial o total de instalaciones, como el Centro de Salud, el bachillerato, el preescolar, la Ayudantía Municipal y el Comisariado, entre otras. Además de la infraestructura, a nivel socioemocional la afectación fue de gran magnitud.

En el momento mismo del terremoto, las experiencias de confusión, inquietud, ansiedad, desesperación y terror fueron generalizadas, y en días posteriores se manifestaron miedo, intranquilidad, preocupación, depresión, ansiedad, incluso en algunos casos falta de sentido de vida. Por otro lado, durante semanas se detuvieron las actividades cotidianas y socioeconómicas, pues la comunidad entera entró en una pausa necesaria. No solo las familias con daños en sus viviendas se vieron afectadas, sino también la población en general.

Si bien acudieron voluntarios de muchas regiones para solventar la crisis, figuras de autoridad e instituciones se tuvieron que replantear una dirigencia que les rebasaba, porque sabían que sanar las grietas era más que reconstruir físicamente los muros. Se dejaron entrever situaciones que debían cambiar, "pliegues" dentro de las instituciones que debían posibilitar análisis y cambios.

Primeramente, se dio la oportunidad de apoyar desde el acompañamiento emocional con intervención en crisis. Un equipo de trabajo del grupo Terapeutas por Morelos empezó a visitar Valle de Vázquez unas semanas después del terremoto. Llegó mucho apoyo en donaciones (sobre todo ropa y alimentos) y dos fundaciones estaban colaborando para evaluar la posibilidad de reconstruir viviendas, pero la situación socioemocional no había sido considerada como una prioridad. En este sentido, la intervención en crisis fue una labor que se pudo realizar durante tres meses con plena aceptación de la población donde las atendidas fueron en su mayoría mujeres.

En el equipo surgió, entonces, la inquietud de continuar el apoyo, en específico con las mujeres, más allá de la intervención en crisis. La situación de vulnerabilización instituida por el contexto sociohistórico, y ahora visibilizada en las fisuras del terremoto, se planteó como una realidad que afectaba en mayor medida a las mujeres rurales en general. La acelerada rutina de su cotidianidad, relegadas al trabajo en sus hogares, impide que las mujeres puedan convivir, organizarse o tener oportunidades de nuevos aprendizajes para crecer. Retomamos el concepto "vulnerabilización" (propuesto por Fernández, 2005), y no "vulnerabilidad", para recalcar la referencia a procesos conformados por estrategias biopolíticas instituidas y no a una situación social que simplemente caracteriza a un sector de la población.

A la situación de vulnerabilización de las mujeres en la comunidad se suman límites que se imponen en los espacios de decisión comunitaria y el estado de pobreza en general que padecen ellas en el campo mexicano. En Valle de Vázquez solo cinco de cien ejidatarios son mujeres y, al igual que en la mayoría de las poblaciones rurales de nuestro país, nunca ha habido una ayudante municipal mujer. Las estadísticas nacionales nos indican que seis de cada diez mujeres rurales son pobres. Además,

Ramírez. Intervención educativa tras el terremoto 2017: comprendiendo procesos identitarios en mujeres rurales Sinéctica 55 www.sinectica.iteso.mx 
comparada con la de las ciudades, la pobreza extrema en las mujeres rurales se da en un 35.2\%, mientras que en el ámbito urbano, en un 15\% (Coneval, 2012; Costa, en IICA, 2018).

La mujer rural está incorporada tanto a los procesos de producción como a los de reproducción, por lo regular sin percibir ingresos económicos suficientes para su manutención o la de su familia. En estas actividades produce, elabora, vende, prepara alimentos, además de las tareas regulares del cuidado de los hijos y la atención a su pareja, familia ampliada y animales, lo que multiplica su jornada. Sus problemas de salud, educación y empleo difieren, en gran medida, de los de las mujeres urbanas (Costa, 1995).

Algunos factores relacionados con estos problemas son salud (aspecto sexualreproductivo, desnutrición, exceso de trabajo físico, carga emocional, entre otros), esperanza de vida (menor que en las zonas urbanas), escolaridad (menor que en las zonas urbanas y menor que los hombres) y migración (esta última como opción para salir de la situación de pobreza). Esto se da en un contexto de dominación que se visibiliza en la cotidianidad en la que predominan formas de ejercer el poder desde múltiples factores (género, clase social, raza, ruralidad) que originan desigualdades.

De ahí la importancia de retomar la visión de interseccionalidad como perspectiva teórica y metodológica en este estudio para comprender las relaciones interpersonales, incluyendo las de género, desde la multiplicidad y las diferentes estrategias instituidas que incrementan las desigualdades. Concepto acuñado por Crenshaw en 1989, la "interseccionalidad" ha sido utilizada históricamente en relación con lo jurídico y desigualdades concretas; sin embargo, puede aplicarse a procesos de desarrollo como el que se llevó a cabo con el grupo de Valle de Vázquez, ya que se abordan cuestiones tanto microsociales (efectos de las estructuras de desigualdad social en las vidas individuales) como macrosociales (implicación de los sistemas de poder en las desigualdades) (Viveros, 2016).

Experiencias exitosas en América Latina han revelado que la intervención en crisis ante desastres socionaturales ha dado resultados valiosos en el establecimiento de lazos entre los pobladores a fin de procesar el trauma, favorecer la salud mental y organizarse para la reconstrucción y prevención de riesgos. En México se ha aplicado en eventos como inundaciones en Chiapas, Guerrero y Michoacán (Castillo, 2014; Álvarez-Gordillo, Álvarez-Gordillo, Eroza-Solana y Dorantes-Jiménez, 2008; Verduzco, Cuevas y Serratos, 2012). En Chile también se realizaron proyectos de intervención tras el terremoto/tsunami de 2010 (Grandón et al., 2016; González-Muzzio, 2013; Concha-Saldías, Micheletti, Olivares-Larín y Rasse-Figueroa, 2015; Imilan y Fuster, 2014; Espinoza, Espinoza y Fuentes, 2015).

En general, hay varios estudios referidos a normativas internacionales, manuales de apoyo en salud mental, estudios concluyentes de instituciones y organizaciones dirigidas a la prevención de futuras crisis socioambientales, la mayoría de las cuales valoran la perspectiva de género (Rodríguez, Zaccarelli y Pérez, 2006; INM, 2013; Drolet et al., 2015; Molina, 2013; Huairou Commission, 2014).

En la revisión de propuestas e investigaciones sobre desastres socionaturales (diecisiete documentos/artículos revisados), dos de ellos tratan proyectos participativos, 
sustentables y que se acercan a un proceso educativo que favorece la estabilidad socioemocional. Fuentes y Ugarte (2015) abordan una experiencia de arteterapia y educación popular en la población de Chaiten, Chile (comunidad desaparecida casi por completo luego de una erupción volcánica en 2008); Rivera, Velázquez y Morote (2014) hacen el análisis de una intervención comunitaria en varias poblaciones de Chincha, Perú, luego del terremoto de 2007. El enfoque participativo y de fortalecimiento comunitario, en este último, permite valorar la forma de intervenir como una propuesta que favorece la sustentabilidad de este último.

Sin embargo, la mayoría de las investigaciones revisadas se limita al acompañamiento durante la etapa de crisis, reconstrucción de infraestructura y económica y prevención de riesgos. A pesar de que la Organización de las Naciones Unidas para la Educación, la Ciencia y la Cultura (Unesco) reconoce desde 1976 el papel que cumple la comunidad en el aprendizaje y la educación, y lo reafirma en la declaración de Beijing 2013 (Yorozu, 2014), al motivar a que se preste atención especial a los grupos vulnerables desfavorecidos, en general hay un vacío en investigaciones relacionadas con la temática educativa posterior a una crisis de tipo socionatural y un cuestionamiento sin resolver acerca de cómo trabajar estos procesos a nivel comunitario y no formal.

Este artículo presenta los avances de una investigación cuyo objetivo es profundizar en los procesos identitarios de las mujeres de Valle de Vázquez después del terremoto de 2017 a partir de una intervención educativa; este enfoque particular de educación no formal se llevó durante más de dos años con un grupo formado por mujeres de la comunidad, quienes de manera voluntaria aceptaron participar en el proceso en el cual la investigación jugó un papel central.

En contra de la visión reduccionista que nos lleva a creer que la escuela es el espacio exclusivo de la educación, la educación no formal considera que el aprendizaje se puede dar por procesos cotidianos desde la familia y la comunidad. Se trata de plantear situaciones en las que fluya el diálogo para identificar necesidades comunes y resolver aspectos que incumben a todos los participantes; espacios que generan autoconfianza, desarrollo de capacidades, conocimientos, decisiones y acciones a favor del bienestar de la misma comunidad. Ahí el trabajo horizontal y el liderazgo múltiple se dan para que exista un aprendizaje de todos. Como afirma Requejo (1994), la educación no formal favorece el desarrollo comunitario, relacionado con “... el proceso de autoayuda, de transformación de la propia comunidad en la identificación y expresión de sus necesidades, orientado todo hacia una mayor responsabilidad y control para promover su propio desarrollo" (p. 62).

El enfoque para trabajar la educación no formal que se utiliza en nuestra investigación es la intervención educativa. También, retomamos algunos elementos de la investigación acción participativa. La lógica del aprendizaje se lleva a cabo planteando acciones (que pueden ser situaciones lúdicas, creativas o dinámicas), las cuales derivan en reflexiones. Surge ahí un diálogo de saberes que se concreta en decisiones y acciones prácticas a favor de las mujeres en lo individual y colectivo. "Los saberes que se construyen son saberes experienciales, referidos a las vivencias de los participantes en su relación con el mundo, así como con saberes organizacionales, referidos a los modos de hacer de una organización" (Flores, 2014).

Ramírez. Intervención educativa tras el terremoto 2017: comprendiendo procesos identitarios en mujeres rurales Sinéctica 55 www.sinectica.iteso.mx 


\section{CONSIDERACIONES METODOLÓGICAS}

Nuestra investigación e intervención educativa y psicosocial parte de una perspectiva interpretativa que permite las conexiones entre lo individual y lo colectivo, entre las experiencias, las decisiones y las acciones, y establece, como diría Corcuff (1995), "puentes entre lo objetivo y lo subjetivo o lo colectivo y lo individual [...] concediendo cierto predominio a las estructuras sociales y a los aspectos macro-sociales de la realidad, al tiempo que integran de diversas formas las dimensiones subjetivas e interaccionales" (p. 23).

La investigación y la intervención educativa se van dando a la par. La investigación produce conocimientos que favorecen la intervención educativa a la vez que brinda elementos para entender el contexto sociohistórico y su impacto en la subjetividad y la vida cotidiana de las mujeres rurales. La intervención busca identificar y reconstruir el tejido social en un proceso de formación grupal, que produce la transformación individual en las participantes, en el grupo de mujeres que se ha formado y en la comunidad misma. La intervención también genera diversos conocimientos, no solo sobre la experiencia de las mujeres y sus identidades, sino de estrategias formativas y pedagógicas dentro del ámbito comunitario.

Si bien el término intervención educativa ha sido utilizado en su mayoría como sinónimo de programas o proyectos educativos, el concepto se refiere a los procesos de formación "... en el que se articulan las experiencias prácticas con acciones de reflexión, análisis y producción teórico-metodológica” (Negrete, 2010, p. 43).

A fin de favorecer un proceso grupal, consideramos que la intervención educativa es la metodología apropiada para este estudio, pues trata de propiciar espacios alternos, no institucionalizados, donde se pueden abordar situaciones cotidianas, problemáticas del ámbito rural y del diario vivir de las mujeres que participan para transitar hacia la introspección, la comunicación y poder tomar decisiones colectivas que las beneficien a ellas y a su comunidad.

Como metodología de educación no formal, la intervención educativa actúa en procesos de emergencia social (como lo es la situación de una comunidad después de la crisis de un terremoto) y permite a la población tomar conciencia de sus necesidades y problemáticas a partir de dispositivos que facilitan la reflexividad y el cambio. Al respecto, Negrete y Peña (2016) afirman que "la intervención educativa toma como punto de partida para sus acciones los enunciados temáticos o problemáticos emitidos por la población con la que se va a trabajar para reconocer en sus preocupaciones, intereses y deseos, las demandas en las que se observará el interjuego de lo instituido y lo instituyente" (p. 374).

En nuestro estudio, "lo instituido" son las creencias y normas que las instituciones mismas instauran desde un sistema patriarcal y paternalista, tradiciones de las cuales se crea dependencia en las personas. Son dos elementos que conviven uno con otro en este círculo vicioso: el paternalismo o asistencialismo de parte de las instituciones, y la dependencia que se fomenta desde temprana edad para asumirse como un modo tradicional y cultural de ser en esta población. Remedi (2015) explica las "lógicas instituidas: su presencia, su fuerza, las formas como tejen la cultura institucional, sin dejar de contemplar y, por tanto, observar que en toda práctica instituida hay procesos que reconocemos como instituyentes, procesos que se están produciendo, gestando en un devenir de nuevas prácticas" (p. 285). 
En gran medida, la fortaleza de las mujeres de Valle de Vázquez hace que nutran y mantengan vivo a este pueblo; por ello, un espacio formativo de reflexión individual y grupal puede promover el análisis crítico a lo instituido para que surja el potencial que empieza expresarse, y que las respuestas y soluciones a las problemáticas cotidianas no tengan que esperar a ser resueltas por otras instancias. Las situaciones instituyentes, en este caso, las posibilidades de autonomía y autogestión para la reconstrucción integral, tanto de las mujeres mismas como de su comunidad, se van dando desde las condiciones y contexto de lo instituido, pues "ambas fuerzas coexisten en la institución" (p. 284).

Rieiro (2009) nombra estas fuerzas implicadas como "dominación" y "resistencia". Explica que, por un lado, existe la fuerza de la dominación, es decir, los mecanismos históricos, sociales o psicológicos que se imponen (que en el caso concreto de Valle de Vázquez estarían relacionados con aspectos instituidos de ruralidad, género y clase social) y, por otro, hay la capacidad de resistencia que se construye a partir del análisis y el diálogo intersubjetivo. Afirma que dentro del mismo sujeto coexisten estas fuerzas que deben ser analizadas, es decir, que tanto la dominación como la resistencia se dan dentro de las propias estructuras sociales de manera inseparable del sujeto.

En Valle de Vázquez, las formas de dominación se instauran y observan en la cotidianidad de las mujeres que las han recibido como tradiciones "naturales" y las van transmitiendo a las nuevas generaciones. Mujeres atrapadas en estos modos de dominación con dificultades para tomar decisiones, como estudiar o trabajar, incluso cuando la situación de pobreza se impone. Es el caso del siguiente testimonio: "Yo estaba por aliviarme de la tercera niña y no teníamos ni para comer [...]. A él se le murieron bastantes vacas y por eso se vino un tiempo difícil. Él es de los que quiere controlar todo. Más antes yo quería trabajar, pero él no me dejaba. Él dice ‘yo te tengo que mantener' [...] como al tiempo de antes, el hombre debe mantener a la mujer" (EI 026).

Al mismo tiempo, junto con esta dominación, se vislumbran pequeños indicios de resistencia: "Y ahorita mi hija estudiando, y vamos al día [...] y le digo que quiero trabajar, y me dice que no. Y me dicen, 'pues no le digas'. Y no le dije, y fue cuando vendía los bolis y eso [...] y sí se enojó porque se enteró [...] pero al menos yo traía verdura o traía otras cosas" (EI 026).

La coexistencia de la dominación y la resistencia es analizada desde las múltiples y sutiles formas de resistir, como el testimonio anterior, que señala que trabajar sin tener que pedir permiso es un paso hacia la posibilidad del cambio, pues “... no se niega la existencia de mecanismos de dominación (de orígenes históricos, sociales, psicológicos) como constituyentes del propio sujeto; sino por el contrario, supone rediscutirlos rescatando también la capacidad de resistencia y autonomía" (Rieiro, 2009, p. 273).

Metodológicamente, ¿cómo pasar de "lo instituido" o fuerzas impuestas por la dominación (paternalismo, dependencia) a "lo instituyente" o fuerzas basadas en la resistencia (conciencia, decisión, organización, autogestión)? En este caso, es un proceso que se puede dar observando las grietas mismas que han dejado no solo los sismos de la tierra, sino la vida de Valle de Vázquez, generación tras generación. En 
estas grietas, donde pareciera resquebrajarse todo, empieza a florecer un verdor renaciente: “... espacios institucionales en que sería posible trabajar: estrechos, hendiduras, grietas, intersticios en los que había poco oxígeno, y que requerían por lo tanto precisión y rapidez en la modalidad de operación e inventiva y originalidad..." (Fernández, 1991, p. 141).

Los intersticios en Valle de Vázquez son la posibilidad de renacer ante la adversidad. La crítica a lo institucional surge desde esta energía interna, un despertar desde el resquebrajamiento interno, primero desde dentro, con base en las identidades mismas de las mujeres, y luego trabajar hacia afuera con las otras mujeres del grupo para, finalmente, favorecer a la comunidad misma.

Surge una fuerza que se expande desde sí hacia los otros y para el colectivo, y lo instituido se mueve para ir dando lugar a lo instituyente. La intervención educativa es el enfoque que queda en medio de esta tensión entre instituido e instituyente, en la que las temáticas se entretejen con los saberes en dinámicas intersubjetivas. Es un proceso formativo que se da a partir de los intersticios, un espacio de acompañamiento donde se establecen situaciones que mueven cuerpos y reflexiones a nivel subjetivo y colectivo para propiciar cambios significativos.

En el aspecto metodológico, elementos de la investigación acción participativa complementan el trabajo de indagación, análisis y cambio en la investigación e intervención educativa. Este enfoque, en el que se escucha la voz de los participantes y facilita el que observen y evalúen una situación o problemática para posibilitar cambios sociales, brinda elementos importantes al proceso. Fals Borda (citado en Montero, 2012), sociólogo y educador colombiano, decía que era un modo de estudiar y de intervenir en la realidad para transformarla. Algunos elementos de la investigación acción participativa que están presentes en este estudio son:

-Una observación participante colaborativa, en la cual las observaciones de las mujeres del grupo son tan valiosas como las de la investigadora o las del equipo de apoyo. Reflexiones escritas en sus cuadernos y participaciones verbales durante las sesiones son la base para analizar las observaciones del grupo.

- Los conocimientos locales y de la vida cotidiana de las mujeres que participan, además de la visión fundamentada de las profesionales que acompañan el proceso.

-Un aprendizaje co-generativo que incorpora conocimientos locales y resuelve situaciones de una mejor manera que la que haría un solo investigador. Las observaciones y colaboración del equipo de apoyo, así como las voces de las mujeres y colaboradores, son fundamentales en la comprensión de la realidad y en las acciones de cambio.

- La validez de los resultados se confirma en las decisiones y acciones realizadas, por ejemplo, en los cambios a nivel subjetivo y en las actividades que se trabajan grupalmente, a la vez que se fundamentan en criterios teóricos.

En cuanto a las técnicas utilizadas en el proceso, las principales son las sesiones grupales de intervención educativa, la observación participante y las entrevistas semiestructuradas profundas. A partir de ellas, surgen narrativas de las mujeres que

Ramírez. Intervención educativa tras el terremoto 2017: comprendiendo procesos identitarios en mujeres rurales Sinéctica 55 www.sinectica.iteso.mx 
se complementan con el registro fotográfico de las sesiones grupales, con las reflexiones escritas de algunas de las participantes y con las entrevistas semiestructuradas realizadas a algunas figuras de autoridad en la comunidad, elementos que agregan información valiosa para la investigación.

Las estrategias de esta investigación responden a los enfoques metodológicos antes explicados, por lo que la mayoría de las técnicas utilizadas se basan en un paradigma que permite que el carácter colectivo esté presente. Las técnicas participativas facilitan la generación de conocimientos y la revisión grupal de experiencias, recursos y necesidades. A través de las sesiones, entrevistas y observaciones, las mujeres participan y comparten testimonios que son evidencia de experiencias, reflexiones y tomas de conciencia de su situación y del proceso.

La observación participante es la herramienta ideal para recoger información en un proceso de investigación-intervención, pues tiene un carácter abierto: el investigador puede cambiar el foco de atención según las narrativas o acontecimientos que se van dando en el momento e implica relaciones con las personas y situaciones observadas. Montero (2012) explica que "participar" en la observación supone que el investigador se coloque en la vida cotidiana de las personas observadas, y tenga cercanía con la situación. También tiene que ver con observarse en la situación como sujeto participante de ella. Esta doble observación -la de las personas y la de sí mismo- debe efectuarse desde una actitud empática, de comprensión y curiosidad científica "a través de la mirada horizontal que se refleja en los ojos del otro" (p. 206).

En las sesiones grupales, la observación participante se realizó tanto por la investigadora como por las otras tres facilitadoras del equipo de apoyo, quienes en cada sesión se rotaron la tarea de registrar las observaciones con anotaciones en una libreta. Asimismo, el compartir de las observaciones en equipo al terminar cada sesión fue fundamental para tener una visión amplia y detallada de lo acontecido. Teniendo como base este material, fue posible elaborar el diario de campo al final de cada jornada de trabajo con descripciones pormenorizadas, observaciones, análisis e interpretaciones de las situaciones dadas.

Otra técnica empleada, la entrevista semiestructurada profunda (que es flexible, dinámica, no directiva, no estandarizada y abierta) facilitó los encuentros con siete mujeres del grupo que pudieron brindar información a través de una conversación en un ambiente de confianza debido al rapport establecido en la etapa inicial del proceso. Seleccionamos a las entrevistadas por ser las más constantes en asistencia y participación en el grupo durante la fase inicial, periodo en el que hicimos las entrevistas.

El procesamiento y análisis de la información inició luego de haber transcrito la información de las entrevistas semiestructuradas, las sesiones grupales y las reflexiones escritas. De esta manera, pudimos contar con una visión general de la información con base en narrativas reordenadas con la herramienta tecnológica Atlas. ti 7, un software que facilita el análisis de los datos cualitativos al procesar documentos primarios desde la organización de una codificación.

Las unidades de análisis consideradas están ligadas a las preguntas de investigación y se plasman en los diferentes momentos del proceso para determinar categorías generales:

Ramírez. Intervención educativa tras el terremoto 2017: comprendiendo procesos identitarios en mujeres rurales Sinéctica 55 www.sinectica.iteso.mx 
- Condiciones de vida: experiencias de las mujeres de Valle de Vázquez en sus historias particulares y en su vida cotidiana en cuanto a actividades, relaciones, historia personal y contexto socioeconómico.

-Terremoto: situaciones relacionadas con el terremoto del 19 de septiembre de 2017, durante y en los meses posteriores a este (en lo emocional, económico, social, ético), que implican experiencias particulares y comunitarias que han tenido las mujeres.

-Intervención educativa: situaciones referidas al proceso formativo a partir de la conformación del grupo de mujeres. Incluye momentos clave de este proceso que tienen que ver con reflexiones, decisiones y tomas de conciencia de las mujeres, tanto en lo individual como grupal.

\section{MOMENTOS CLAVE DE UNA INTERVENCIÓN EDUCATIVA CON MUJERES}

Haciendo un recorrido de la investigación e intervención educativa, esta tuvo su punto de partida con la entrada al campo, cuyo objetivo fue familiarizarse con la comunidad e identificar sus necesidades. Inició en octubre de 2017 con la intervención en crisis por parte del grupo Terapeutas por Morelos. Asistimos cuatro interventoras con visitas de apoyo a la población al considerar que era un momento necesario para acompañar en lo psicoemocional, aspecto que no había sido considerado dentro de las prioridades institucionales. Surgió, entonces, la intención de llevar a cabo la investigación e intervención educativa.

La intervención en crisis concluyó en diciembre de 2017 y el equipo completo continuó la labor de apoyo en las fases siguiente. Entre enero y marzo de 2018 se dio la exploración e indagación de la situación de los damnificados y de la comunidad en general, así como la coordinación del apoyo mutuo con la Escuela Secundaria Técnica "Lorenzo Vázquez" (que posteriormente brindó un espacio físico para las primeras sesiones de intervención educativa). También fue el momento de realizar el análisis de la información para determinar las líneas y preguntas de la investigación e intervención educativa. En estos primeros meses logramos establecer lazos de comunicación y confianza con los pobladores; fuimos trabajando en la revisión de estudios e investigaciones con temáticas similares (estudios de género, desastres socioambientales, ruralidad), además de armar el proyecto y reconocer el territorio-población.

Después de haber identificado necesidades y recursos de la población a partir del acercamiento, las entrevistas informales y las reuniones con algunas autoridades de la comunidad, empezó el segundo momento del proceso formativo, es decir, el trabajo colectivo desde la intervención educativa con el grupo de mujeres.

Las reuniones grupales se dieron a partir de abril de 2018, empezando con la sesión informativa sobre lo que se trabajaría con las mujeres. En esta, además de dar a conocer los objetivos de la investigación e intervención e indagar acerca de las inquietudes de las mujeres desde una actividad lúdica-reflexiva, aplicamos el cuestionario sociodemográfico, cuyos resultados fueron importantes para el diagnóstico. De las 49 mujeres que asistieron a la reunión llevada a cabo en la secundaria, aproximadamente15 decidieron integrarse a las sesiones semanales. Es importante 
señalar que no siempre acudieron las mismas mujeres, ante todo al cambiar las sesiones de lugar, pues algunas de ellas dejaron de asistir y otras se integraron y pasaron a formar parte vital del grupo y del proceso.

Desde un acompañamiento constante, los temas fueron emergiendo con el reconocerse como mujeres y como grupo inserto en una comunidad y, desde el diálogo, también los sueños colectivos se empezaron a construir. La apertura a otras miradas se fue volviendo parte de la dinámica (la de las otras participantes, la de lo comunitario y también la de las interventoras). Lo lúdico y la expresión verbal y corporal fueron herramientas básicas en el devenir del proceso, pues favorecieron que las acciones y reflexiones fluyeran con la participación abierta de las mujeres.

El grupo de mujeres y el equipo de interventoras se fue integrando, en un primer momento, a partir de talleres de autoconocimiento con algunos temas que surgieron de sus necesidades: "El sentido de mi nombre", "El valor de mi cuerpo", "Mis fortalezas" y "Mi autoestima". En estas sesiones, el grupo fue trabajando el autoconocimiento y la capacidad de introspección, a la vez que se dio el reconocimiento de la otra, pues se abrieron espacios para que se escucharan entre sí. De lo lúdico, de lo que compartieron, los relatos y las reflexiones que fueron anotando en sus cuadernos, los temas surgieron y se desarrolló una experiencia común. "Durante el despliegue del proyecto, lo que marca la secuencia de las acciones es la propia acción con la población intervenida, los encuentros realizados nos van guiando en lo que sigue" (Negrete y Peña, 2016, p. 379).

A continuación, presentamos las situaciones y los momentos clave que permitieron el despliegue de la investigación e intervención educativa:

- El tema del terremoto fue un eje que permitió el surgir del grupo, lo cual lo cohesionó. El 19 de septiembre de 2017, muchas mujeres de Valle de Vázquez se encontraban en sus hogares, atareadas con la rutina diaria, aisladas, de alguna manera, de las vecinas y del resto de la comunidad. Aquí el testimonio de una entrevistada:

Yo estaba tendiendo ropa, cuando oigo el ruido [...] y en vez de correr al corral me vine para acá adentro [...], me asusté bien feo. Yo pensé que era el fin del mundo, y corro para acá, y me asomo y no veo nada, nada. Y me vuelvo a regresar [...] y ya no me dio tiempo de salir, y me quedé en la puerta temblando, asustada. Estaba yo solita, y yo gritaba: “Estoy sola! Dios mío ¡estoy sola!”... (EI 017).

A un año del terremoto, en el grupo se efectuó una actividad profunda que las conectó con la energía de la tierra. Luego de recordar el evento, cada una de las participantes sopló sus miedos y preocupaciones a un puñado de semillas (mezcla de maíz, frijol y arroz) para luego enterrarlas en la tierra. Compartieron los sentimientos que aún permanecían en sus cuerpos y los soltaron; la conexión se observó más allá del intercambio de comentarios, al hacerse evidente la conexión con la tierra, la cual se volvió a valorar como algo que podía ser estable y ser un espacio fértil a futuro. Las mujeres empezaron a concebirse como parte de una misma experiencia.

La experiencia dos años después del terremoto reflejó una mayor madurez del grupo. Organizamos una actividad con una conexión más profunda con sus identidades, en la que, entre todas, se sembró una rosa. Esto ayudó a resignificar las experiencias, pues, al enfocarse no en los miedos o en las pérdidas, sino en lo mucho 
que han crecido y en los cambios que han tenido, se reafirmaron en la posibilidad de construir un futuro en común desde proyectos concretos.

- La participación y el entusiasmo se percibió desde las primeras sesiones de intervención. Las mujeres llegaron voluntariamente, se entregaron a las actividades y permitieron que el aprendizaje compartido se fuera dando. En la temática sobre el autoconocimiento del cuerpo, abordada en la segunda sesión grupal, surgieron decisiones importantes que se fueron verbalizando en el grupo. Se habló de comer sano, de hacer ejercicio y valorar la belleza personal. A raíz de esto, algunas de las participantes decidieron reunirse a hacer ejercicio. A pesar de que no eran la mayoría, invitaron a más vecinas (algunas se integrarían luego al grupo) para hacer zumba todas las tardes en casa de una de ellas.

- Después de estar reuniéndose durante los primeros cuatro meses en la secundaria de la comunidad, las mujeres decidieron cambiar el sitio de reunión. El lugar, como espacio instituido, se percibía por ellas como algo que las limitaba (el director y los maestros de la secundaria estaban al pendiente de las actividades que se realizaban en el grupo, escuchaban tras la puerta y se asomaban por las ventanas del salón que habían prestado para las sesiones). Ellas propusieron hacer la reunión en un lugar más cerca del centro del pueblo, por lo que una de las participantes ofreció su casa.

Al cambiar de lugar, se abrieron posibilidades desde un espacio que movilizó y generó cambios. El proceso, entonces, empezó a ser apropiado por el grupo, pues si bien el espacio era la casa de una de las participantes (la cual tiene un liderazgo importante en el grupo), de alguna manera, se volvió de ellas. También permitió que nuevas participantes se acercaran para aprovechar la oportunidad de unirse al grupo (mujeres que -a pesar de que la invitación era abierta a toda la población- quizá sentían que era un espacio exclusivo para madres de hijos en la secundaria). Al grupo se incorporaron madres jóvenes y también mujeres de la tercera edad, lo que lo volvió heterogéneo en cuanto a edad, escolaridad y experiencia. Pudimos constatar que, al salirse de la secundaria, se empezó a jugar con la estructura, una especie de liberación que implicó que el grupo pasó a ser un "nosotros". También, ayudó a quitar la sensación de que iban a "clases" y empezaron a percibir que era un espacio de convivencia y reflexión.

- Se fue visibilizando lo que estaba sucediendo a nivel individual, a la vez que se fue liberando la fuerza del grupo; se generó claridad en pensamientos, sentimientos y acciones; se abrió un espacio para hacer tomas de conciencia, un espacio de creación conjunta, pues, a partir de actividades lúdicas y verbalizaciones, sus sabidurías fueron dialogando para afianzar al grupo. Al descubrir su voz en el grupo, cada una de las participantes fue recuperándose para "ser" en otros espacios más allá del grupo mismo, ya que, cuando narraban anécdotas o fragmentos de su historia personal o familiar, reafirmaban sus identidades y, a la vez, se iban identificando en historias comunes que se entrelazaban. En una reflexión escrita, una participante expresa:

Estas sesiones me gustan porque tenemos un tiempo para vernos desde otro punto de vista. Yo soy muy reservada y al estar en grupo me gusta compartir mis vivencias con las demás y me da mucha seguridad y confianza en mí. Para mí es una satisfacción participar y ser parte de este grupo porque aprendo a socializar y a entender a las demás personas. Ponernos en los zapatos de las otras es favorable para nuestra persona. También me enseña a valorarme ya que a veces me sentía un poco inferior a las demás por mi forma de ser o actuar. El contar nuestras experiencias en grupo me hace un poco más libre para convivir mejor (R 026).

Ramírez. Intervención educativa tras el terremoto 2017: comprendiendo procesos identitarios en mujeres rurales Sinéctica 55 www.sinectica.iteso.mx 
- A seis meses de estar participando en la intervención, el grupo se observó en su caminar y, con una actividad de armar una línea del tiempo con fotografías de todas las sesiones, visualizó el recorrido andado. Cada participante expresó lo vivido, los momentos significativos, los puntos de quiebre a nivel individual y grupal. Al verse en un proceso, se concibieron como un grupo y se nombraron. Entre todas fueron mencionando características y, al final, se describieron: "Somos un grupo de mujeres valientes, sobrevivientes, esforzadas, que hemos trabajado en equipo sobre el valor de nosotras como mujeres" (S012).

- Surgió la inquietud de definir qué querían como grupo, y comprender que no había conocimientos teóricos que "aprender", sino metas a alcanzar entre todas. Las mujeres decidieron reunirse por su cuenta (por primera vez) y dialogaron entre ellas sobre lo que querían. Surgió una lluvia de ideas de actividades productivas, así como la intención de seguir discutiendo temas de autoconocimiento y relaciones interpersonales. Se evidenció que el grupo seguía teniendo la necesidad de ser acompañado en su desarrollo humano y, a la vez, se reconocía en sus capacidades introspectivas, creativas y productivas. En ese momento, observamos al grupo cohesionado, al empezar a definir un autoconcepto y un sentido colectivo.

- En un momento dado, el grupo sorprendió al equipo de interventoras, pues, al llegar a la sesión acordada, ellas se habían reunido una hora antes para compartir recetas de repostería y preparar unas donas entre todas, las cuales, al final de la sesión, se compartieron en una alegre convivencia. Decisiones como esta y como la de hacer ejercicio diariamente fueron indicios fuertes de una autonomía de grupo.

- La lluvia de ideas se fue poniendo en práctica, revisándose una y otra vez, hasta llegar al planteamiento concreto de proyectos productivos que empezaron a desarrollarse. El grupo se fue organizando, definiendo exactamente qué quería hacer desde una flexibilidad en la que, si no todas estaban de acuerdo, se valía diversificarse. Concordaron en que las sesiones quincenales con el equipo serían las que les uniría $\mathrm{y}$, aparte, empezarían a realizar dos actividades productivas: manualidades y huerto (no todas participaron en ambos proyectos).

- Las temáticas abordadas en relación con la comunicación permitieron que la voz de cada una de ellas fuera moderándose y siendo más puntual (en el caso de quien naturalmente se expresaba con tendencia impulsiva y directa) o emergiendo de un silencio profundo (en el caso de quien se había sometido, aguantando sus ideas y sentimientos, porque "así le enseñaron a ser"). Surgieron decisiones como, por ejemplo, la de hablar con el director de la secundaria ante una situación que percibían como injusta para reclamar sus derechos. Estas iniciativas se dialogaron, se practicaron y se llevaron a cabo como experiencias de autonomía que fueron mejorando sus vidas y las de sus hijos.

- Por iniciativa de ellas, se dio una evaluación constante del andar del grupo y del compromiso a nivel individual. Periódicamente, se revisaba cómo estaba el grupo, qué faltaba, qué esperaban, qué podían y qué no podían hacer, a qué se comprometían y a qué no. Así, al cabo de año y medio del proceso se hizo una recapitulación de “¿cómo llegué al grupo?”, “cómo estoy/estamos hoy?” y “¿cómo me veo/cómo veo al grupo en un año?". Cada una pasó a escribir sus sentimientos e intenciones en carteles que fueron colocados para observar el proceso completo. La visualización 
de un proyecto futuro de grupo fue evidente y, a pesar de que habían expresado también la sensación de querer que el equipo permaneciera, había conciencia de que el compromiso y la constancia era de ellas. Se visualizaron prósperas, unidas y trabajando juntas en proyectos diversos.

Finalmente, se dio la etapa de cierre del proceso de intervención educativa, momento, como refieren Negrete y Peña, en que "se valoran alcances y limitaciones de las acciones" (2016, p. 380), se observan los cambios en el proceso, así como la posibilidad de continuar desde decisiones que se hayan tomado en el grupo o desde acciones que favorecen a la comunidad. Esta etapa inició en septiembre de 2019 y concluyó en febrero de 2020. Además de los logros en el desarrollo subjetivo e intersubjetivo mencionado en la etapa del despliegue de la intervención educativa, algunos aspectos permitieron ir cerrando el proceso:

- La capacidad de reflexividad sobre los cambios que tuvieron en dos años, en los que fue patente la conciencia de su propio desarrollo como personas a partir de acciones que reafirmaron sus identidades.

- La conciencia y comunicación de su compromiso y de sus límites con relación a los proyectos productivos grupales.

-La capacidad de reunirse como grupo por su cuenta, por ejemplo, y comprometerse tres veces por semana para compartir el bordado, intercambiar saberes y aprender nuevas técnicas en su trabajo de manualidades.

- Las decisiones autosustentables, como la de invertir en materiales para continuar bordando o la decisión de capacitarse y compartir sabidurías en grupo con relación al manejo de plantas para que cada una pueda trabajar su hortaliza en casa.

-La visualización de un proyecto a futuro que da sentido al grupo.

En síntesis, las acciones en la investigación e intervención educativa se van dando a la par; el proceso formativo es el espacio que guía la investigación misma. Este proceso, en el que las sesiones se van evaluando y planificando junto con el equipo de apoyo, no fue previamente determinado, sino que las mujeres mismas, desde sus inquietudes, fueron dando las pautas a seguir. De las sesiones grupales y de las entrevistas individuales, surgieron las narrativas que luego analizamos a partir de categorías que se establecen desde el fundamento teórico-conceptual de este estudio y desde la realidad que fueron expresando las mujeres de Valle de Vázquez.

\section{REFLEXIONES FINALES}

El análisis, tanto de los diálogos grupales como de las narrativas obtenidas en entrevistas individuales, aún se está realizando; sin embargo, los momentos clave descritos son experiencias fundamentales que generan líneas concluyentes en este estudio.

En una población rural como Valle de Vázquez, los procesos identitarios de las mujeres son muy particulares, ya que existe un contexto de dominación y de vulnerabilización que viven (agravada después del terremoto de 2017) que, a su vez, puede ser el cimiento de la misma resistencia y liberación de estas condiciones cuando se plantea un adecuado proceso educativo. 
Las formas tradicionales de asumir los compromisos del hogar (que parten de una sociedad patriarcal) hacen que las mujeres en esta comunidad vivan enfocadas en la atención de sus hijos y sus familiares inmediatos, además de múltiples tareas en el hogar y actividades productivas no necesariamente reconocidas. Se trata de mujeres activas y comprometidas y, a la vez, aisladas por su rutina cotidiana. Esto se evidenció durante el terremoto, pues muchas de ellas, desde sus hogares, vivieron soledad, angustia, preocupación por sus hijos, y otras experiencias paralizantes, por una situación que no pudieron controlar ni compartir con otras.

Después del terremoto, el apoyo externo que recibió la comunidad de Valle de Vázquez no consideró las necesidades reales de la población ni valoró la situación psicosocial de los afectados. Ante eso, el trabajo de intervención en crisis fue importante, pero no suficiente. El terremoto permitió entrever una realidad que debía ser analizada, pues a partir de sus "fisuras" se dieron líneas de fuga para que la población en general, y las mujeres en particular, pudieran atreverse a reunirse, pedir apoyo y empezar a reflexionar sobre su situación de vida.

Así, decidimos dar seguimiento a la labor realizada en los meses de intervención en crisis con el apoyo a un grupo de mujeres, y pusimos el foco en los procesos identitarios individuales y colectivos a partir de las experiencias de las participantes en su vida cotidiana y ante el desastre socioambiental; valoramos que un terremoto es una oportunidad para promover tomas de conciencia y cambios positivos en la comunidad.

Este proceso de investigación e intervención educativa tuvo la intención de comprender la situación después del terremoto de las mujeres en una comunidad rural de Morelos, lo que implicó abarcar la integralidad de una realidad que no solo se impone desde la crisis, sino que viene de un contexto sociohistórico y se expresa en formas de vulnerabilización desde una amplitud de dimensiones.

El espacio que brindó la intervención educativa fue significativo para un grupo de mujeres que fueron creciendo a nivel psicosocial durante el proceso. La educación no formal es una alternativa para las mujeres que viven en zonas rurales, donde la cotidianidad les limita el acceso a formas de convivencia, organización y desarrollo humano. En el caso de Valle de Vázquez, la intervención educativa fue una pausa necesaria en el ajetreo diario de las mujeres, un "alto en el camino" que planteó situaciones basadas en una metodología lúdica y participativa, y permitió espacios de interconexión.

Fueron dos años y medio de sesiones quincenales en un proceso formativo que facilitó, por un lado, la introspección y, por otro, la reflexividad colectiva. En diferentes momentos se revisaron aspectos clave de sus identidades como mujeres, que llevaron a tomas de conciencia y a cambios profundos. El grupo se expresó articulando saberes para ir adoptando decisiones que las impulsó a desplegar su potencial y a empezar a construir nuevas formas de identidad.

Este proceso se dio en tres momentos. Inició en un punto de partida desde la incertidumbre y la escucha activa de la interventora y del equipo de apoyo que se implicaron con las mujeres de la comunidad. La importante conexión originó que, a partir de abril de 2018, se iniciaran las sesiones en las que participaron un promedio de quince mujeres. 
En un segundo momento, desplegamos la intervención a partir de la lógica de que aquello que ha sido construido por mensajes y experiencias vividas en la infancia y la adolescencia, determinado por factores sociohistóricos y culturales que se transmiten de generación en generación, puede también ser deconstruido, sobre todo si se vive colectivamente una crisis circunstancial como la que encierra un desastre socioambiental. La deconstrucción de una aparente estructura sólida de identidad, en un símil al terremoto de 2017, implicó reflexionar, valorar qué piezas individuales y colectivas debían conservarse y cuáles debían transformarse en busca de una mayor estabilidad. Así, el proceso formativo de la intervención educativa brindó un espacio para el cuestionamiento de una realidad atravesada por criterios de género, situación socioeconómica, territorialidad rural y otros factores, donde finalmente empezó la reconstrucción de identidades, una reconstrucción basada en acciones y decisiones desde una conciencia interior, junto con las otras mujeres y para la comunidad.

En la última etapa observamos cambios en el proceso. El grado de autonomía y autogestión era tal que en febrero de 2020 decidimos cerrar la intervención educativa. Las mujeres del grupo empezaban a reunirse alrededor de proyectos productivos gestados por ellas mismas, decidían cuidar de su salud a través de acciones concretas, como reunirse a hacer ejercicio, reafirmaban sus sentimientos y pensamientos expresándose entre ellas y con sus familias, se sentían apoyadas desde una realidad compartida en la que sus identidades se fueron transformando.

Existen procesos identitarios individuales en la mujer rural y también procesos identitarios colectivos. Estos últimos se van conformando a partir de la intersubjetividad. En palabras de Giménez (1997), “... la identidad no es una esencia, un atributo o una propiedad intrínseca del sujeto, sino que tiene un carácter intersubjetivo y relacional" (p. 12). En el caso de Valle de Vázquez, la toma de decisiones respecto a un espacio grupal propio, la caracterización del grupo desde el nombrarse entre todas, la decisión de realizar proyectos productivos en común, entre otros, fueron situaciones que reafirmaron un nuevo modo de identidad colectiva.

Durante el proceso se fue constatando que la posibilidad de resistirse a la vulnerabilización y a la dominación en que vive la mujer rural, reflexionando, compartiendo y realizando pequeños cambios, brinda esperanza en cuanto a su situación. El hecho de visualizarse procesualmente en una situación más autónoma puede ser el motor que mantenga el proceso colectivo, más allá de la investigación e intervención educativa que llevamos a cabo.

Después del cierre del proceso formativo, iniciamos la revisión de narrativas surgidas tanto de los diálogos durante las sesiones como de las reflexiones en las entrevistas semiestructuradas que hicimos a las participantes. Aún está pendiente una revisión minuciosa de categorías que van emergiendo; sin embargo, ya podemos visualizar elementos importantes para comprender cómo ha sido el proceso de construcción de identidades de las mujeres de Valle de Vázquez, así como la deconstrucción de estas que se fue dando en el espacio de intervención educativa.

\section{REFERENCIAS BIBLIOGRÁFICAS}

Álvarez-Gordillo, G., Álvarez-Gordillo, L., Eroza-Solana, E. y Dorantes-Jiménez, J. (2008). Propuesta educativa para la gestión del riesgo de desastres en la región 
de Sierra de Chiapas, México. RMIE, julio-septiembre, núm. 38, vol. 13, pp. 919943. Recuperado de http://www.comie.org.mx/v5/sitio/revista-mexicana-deinvestigacion-educativa/

Castillo, 0. (2014). La construcción social y los imaginarios sociopolíticos del desastre: una reflexión sobre las inundaciones en Zona Diamante. El Cotidiano, septiembre-octubre, núm. 187, pp. 249-263. Recuperado de https://issuu.com/elcotidiano/docs/187

Concha-Saldías, C., Micheletti, S., Olivares-Larraín, R. y Rasse-Figueroa, A. (2015). Reconstrucción post-terremoto en la ruralidad del Maule. Revista Bitácora Urbano Territorial, enero-junio, núm. 1, vol. 25, pp. 71-80. Recuperado de https://revistas.unal.edu.co/index.php/bitacora/article/view/40210

Coneval (2013). Informe de la pobreza en México. Distrito Federal.

Corcuff, P. (1995). Las nuevas sociologías. Construcciones de la realidad social. España: Alianza Editorial.

Costa, N (1995). La mujer rural en México. Recuperado de http://www.pa.gob. $\mathrm{mx} /$ publica/cd_estudios/Paginas/autores/costa\%20nuria\%20mujer\%20 rural\%20en\%20mexico.pdf

Drolet, J. et al. (2015). Women rebuilding lives post-disaster: Innovative community practices for building resilience and promoting sustainable development. Gender \& Development.

Espinoza, A., Espinoza, C. y Fuentes, A. (2015). Retornando a Chaitén: diagnóstico participativo de una comunidad educativa desplazada por un desastre socionatural. Magallanía, núm. 3, vol. 43, pp. 65-76. Recuperado de http:// www.magallania.cl/index.php/magallania/article/view/776

Fernández, A. (2005). Vulnerabilización de los jóvenes en Argentina: política y subjetividad. Nómadas, octubre, núm. 23, pp. 132-139.

Fernández, A. (1991). Laberintos institucionales. Buenos Aires: Búsqueda.

Flores, J. (2014). Aprendizaje comunitario en México. EAD 81. Recuperado de https://www.dvv-international.de/es/educacion-de-adultos-y-desarrollo/ ediciones/ead-812014-comunidades/aprendizaje-comuntiario-en/aprendizaje-comunitario-en-mexico/

Fuentes, A. y Ugarte, A. (2015). Re-creando Chaitén en comunidad: arteterapia y educación popular en desastres socionaturales. Chile: CIVDES.

Giménez, G. (1997). Materiales para una teoría de las identidades sociales. Frontera Norte, julio-diciembre, núm. 18, vol. 9.

González-Muzzio, C. (2013). El rol del lugar y el capital social en la resiliencia comunitaria posdesastre. Aproximaciones mediante un estudio de caso después del terremoto del 27/F. EURE (Santiago), núm. 117, vol. 39. Recuperado de https://www.eure.cl/index.php/eure/article/view/218/586

Grandón, P., Pérez-Salas, C., Rincón, P., Vázquez, J., Cova, F., Panadero, S. y Saldivia, S. (2016). Organización, convivencia y reivindicaciones en tres campamentos de damnificados post terremoto-tsunami del 21/F en Talcahuano, Chile. Ajayu. Órgano de Difusión Científica del Departamento de Psicología de la Universidad Católica Boliviana, marzo, núm. 1, vol. 14, pp. 121-139. Recuperado de https://www.ucb.edu.bo/publicaciones/ajayu/v14n1/ v14n1a06.html

Huairou Commission (2014). Mujeres resilientes. La Plataforma de Practicantes Comunitarias para la Resiliencia. 
IICA (2018). Luchadoras: mujeres rurales en el mundo: 28 voces autorizadas. Costa Rica.

INM (Instituto Nacional de las Mujeres) (2013). Equidad de género en la gestión integral del riesgo. México.

Imilan, W. y Fuster, X. (2014). Llico post 27F: la comunidad invisibilizada en la reconstrucción de su hábitat residencial. En M. Vargas et al. (2014). Ética, poder y territorio. Chile: FACSO.

Molina, E. (2013). Género y resiliencia en la gestión integral del riesgo de desastres (tesis de posgrado). Facultad de Ciencias Sociales, Universidad de Castilla-La Mancha.

Montero, M. (2012). Hacer para transformar. El método en la psicología comunitaria. Buenos Aires: Paidós.

Negrete, T. (2010). La intervención educativa. Un campo emergente en México. Revista de Educación y Desarrollo, abril-junio, vol. 13. Recuperado de http:// www.cucs.udg.mx/revistas/edu_desarrollo/anteriores/13/013_Negrete

Negrete, T. y Peña, A. (2016). La intervención educativa: una respuesta ante las condiciones de emergencia social. En E. Hernández-Vela. Política internacional: temas de análisis. México: UNAM.

Remedi, E. (2015). Un lugar incómodo. Algunas reflexiones en torno a la intervención educativa. En E. Trevino y J. Carbajal. Políticas de la subjetividad e investigación educativa (pp. 283-297). México: Balam.

Requejo, A. (1994). La educación "no formal" de adultos: ciudad educativa y "ciudad sana" en el contexto del desarrollo comunitario y la intervención socioeducativa en la "tercera edad". Teoría de la Educación, núm. 194, vol. VI, pp. 61-76. Recuperado de https://revistas.usal.es/index.php/1130-3743/ article/view/3028

Rieiro, A. (2009). El sujeto: entre las relaciones de dominación y resistencia. Recuperado de https://es.scribd.com/document/378610286/Anabel-RieiroEl-sujeto-entre-relaciones-de-dominacion-y-resistencia-pdf

Rivera, M., Velázquez, T. y Morote, R. (2014). Participación y fortalecimiento comunitario en un contexto post-terremoto en Chincha, Perú. Psicoperspectivas, núm. 2, vol. 13, pp. 144-155. Recuperado de http://departamento.pucp. edu.pe/psicologia/wp-content/uploads/2015/04/Rivera-Velazquez-Morote-2014.pdf

Rodríguez, J., Zaccarelli, M. y Pérez, R. (eds.) (2006). Guía práctica de salud mental en situaciones de desastres. Washington: OPS/OMS.

Verduzco, F., Cuevas, A. y Serratos, E. (2012). El enfoque de género en la etapa de reconstrucción de un desastre: análisis de programas sociales aplicados con equidad de género en Angangueo, Michoacán. Revisa de Estudios de Género. La Ventana, julio-diciembre, núm. 36, vol. IV, pp. 87-132. Recuperado de http://revistalaventana.cucsh.udg.mx/index.php/LV/article/view/697

Viveros, M. (2016). La interseccionalidad: una aproximación situada a la dominación. México: UNAM.

Yorozu, R. (2014). Aprendizaje comunitario en la sociedad del aislamiento. EAD 81/2014. Comunidades. Recuperado de https://www.dvv-international.de/ es/educacion-de-adultos-y-desarrollo/ediciones/ead-812014-comunidades/articulos/aprendizaje-comunitario-en-la-sociedad-del-aislamiento/ 